\title{
INTEGRALS OF PRODUCTS OF HURWITZ ZETA FUNCTIONS AND THE CASIMIR EFFECT IN $\phi^{4}$ FIELD THEORIES
}

\author{
M. A. Shrot, M. P. Chaudhary and R. B. Paris
}

\begin{abstract}
We evaluate two integrals over $x \in[0,1]$ involving products of the function $\zeta_{1}(a, x) \equiv$ $\zeta(a, x)-x^{-a}$ for $\Re(a)>1$, where $\zeta(a, x)$ is the Hurwitz zeta function. The evaluation of these integrals for the particular case of integer $a \geqslant 2$ is also presented. As an application we calculate the $O(g)$ weak-coupling expansion coefficient $c_{1}(\varepsilon)$ of the Casimir energy for a film with Dirichlet-Dirichlet boundary conditions, first stated by Symanzik [Schrödinger representation and Casimir effect in renormalizable quantum field theory, Nucl. Phys. B 190 (1981) 1-44] in the framework of $g \phi_{4-\varepsilon}^{4}$ theory.
\end{abstract}

Mathematics subject classification (2010): 11M35, 11B68, 33B15, 33E20.

Keywords and phrases: Hurwitz zeta function; integrals; Feynman integrals; Casimir energy.

\section{REFERENCES}

[1] J. Andersson, Mean value properties of the Hurwitz zeta-function, Math. Scand., 71 (1992), 295 300.

[2] T. M. Apostol, Introduction to analytic number theory, Springer Science \& Business Media, 2013.

[3] T. Arakawa, T. Ibukiyama, M. KaneKo, And D. Zagier, Bernoulli numbers and zeta functions, Springer, 2014.

[4] F. V. AtKinson, The mean-value of the Riemann zeta function, Acta Mathematica, 81, 1 (1949), 353-376.

[5] M. Bordag, G. L. Klimchitskaya, U. Mohideen, and V. M. Mostepanenko, Advances in the Casimir effect, Oxford University Press, 2009.

[6] M. Burgsmüller, H. W. Diehl, And M. A. Shpot, Fluctuation-induced forces in strongly anisotropic critical systems, JSTAT, 2010 (2010), P11020, Erratum: JSTAT E05001 (2011).

[7] H. B. G. CASIMIR, On the attraction between two perfectly conducting plates, Proc. K. Ned. Akad. Wet., B51 (1948), 793-795.

[8] D. Cvijović AND H. M. SRivastava, Evaluations of some classes of the trigonometric moment integrals, J. Math. Anal. Appl., 351, 1 (2009), 244 - 256.

[9] H. W. Diehl, D. GrÜEnEBerg, AND M. A. ShPot, Fluctuation-induced forces in periodic slabs: Breakdown of epsilon expansion at the bulk critical point and revised field theory, Europhys. Lett., 75, 2 (2006), 241-247.

[10] E. Elizalde, Ten physical applications of spectral zeta functions, Lecture Notes in Physics, vol. m35, Springer, Berlin, Heidelberg et. al, 2012.

[11] O. EsPinOSA AND V. H. Moll, On some integrals involving the Hurwitz zeta function: Part 1, The Ramanujan Journal, 6, 2 (2002), 159 - 188.

[12] On some integrals involving the Hurwitz zeta function: Part 2, The Ramanujan Journal, 6, 4 (2002), $449-468$.

[13] S. R. FINCH, Mathematical constants, Cambridge University Press, 2003.

[14] S. HU, D. KIM, AND M-S. KIM, On some integrals involving the Hurwitz-type Euler zeta functions, arXiv: 1508.04084 (2015).

[15] A. HuRwitz, Einige Eigenschaften der Dirichlet'schen Functionen $F(s)=\sum\left(\frac{D}{n}\right) \cdot \frac{1}{s}$ die bei der Bestimmung der Klassenzahlen binärer quadratischer Formen auftreten, Z. Math. Phys., 27 (1882), 86-101. 
[16] S. Kanemitsu, H. Kumagai, And M. Yoshimoto, Sums involving the Hurwitz zeta function, The Ramanujan Journal, 5 (2001), 1, 5-19.

[17] S. Kanemitsu, Y. TANigawa, And M. Yoshimoto, Structural elucidation of the mean square of the Hurwitz zeta-function, J. Number Theory, 120, 1 (2006), $101-119$.

[18] A. A. Karatsuba And S. M. Voronin, The Riemann zeta-function, Walter de Gruyter, Berlin, New York, 1992.

[19] M. KARDAR AND R. Golestanian, The "friction" of vacuum, and other fluctuation-induced forces, Rev. Mod. Phys., 71, 4 (1999), 1233-1245.

[20] M. Katsurada AND K. Matsumoto, Explicit formulas and asymptotic expansions for certain mean square of Hurwitz zeta-functions, Proc. Japan Acad., 69 Ser. A (1993), 303-307.

[21] _ Explicit formulas and asymptotic expansions for certain mean square of Hurwitz zetafunctions I, Math. Scand., 78 (1996), 161-177.

[22] _ Explicit formulas and asymptotic expansions for certain mean square of Hurwitz zetafunctions II, New Trends in Probability and Statistics, 4 (1997), 119-134.

[23] J. F. Koksma And C. G. LeKKerkerker, A mean-value theorem for $\zeta(s, w)$, Indag. Math. (Proceedings), 55 (1952), $446-452$.

[24] U. Kraemmer and A. Rebhan, Advances in perturbative thermal field theory, Rep. Prog. Phys., 67, 3 (2004), 351-431.

[25] M. KRECH, Casimir effect in critical systems, World Scientific, Singapore, 1994.

[26] M. KRECH AND S. DiETRICH, Free energy and specific heat of critical films and surfaces, Phys. Rev. A, 46, 4 (1992), 1886-1922.

[27] H.-L. Li, M. Hashimoto, And S. Kanemitsu, Examples of the Hurwitz transform, J. Math. Soc. Japan, 61, 3 (2009), 651-660.

[28] K. MATsumoto, Recent developments in the mean square theory of the Riemann zeta and other zetafunctions, Number Theory (R. P. Bambah, V. C. Dumir, and R. J. Hans-Gill, eds.), Birkhäuser Basel, Basel, 2000, pp. 241-286.

[29] F. W. J. Olver, D. W. Lozier, R. F. Boisvert, and C. W. Clark (eds.), NIST handbook of mathematical functions, Cambridge University Press, Cambridge, 2010.

[30] H. M. SRIVAstava AND J. ChOI, Zeta and q-zeta functions and associated series and integrals, Elsevier, Amsterdam, 2012.

[31] K. SYMANZIK, Schrödinger representation and Casimir effect in renormalizable quantum field theory, Nucl. Phys. B, 190 (1981), 1-44.

[32] E. T. Whittaker And G. N. Watson, A course of modern analysis, Cambridge University Press, Cambridge, London, and New York, 1996. 